\title{
An operator description of entanglement matching in quantum teleportation
}

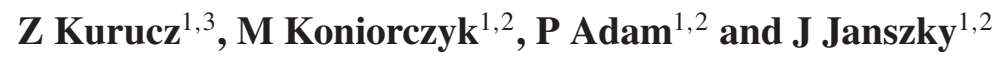 \\ ${ }^{1}$ Research Institute for Solid State Physics and Optics, Hungarian Academy of Sciences, \\ PO Box 49 H-1525 Budapest, Hungary \\ ${ }^{2}$ Research Group for Nonlinear and Quantum Optics, Hungarian Academy of Sciences, and \\ Institute of Physics, University of Pécs, Ifjúság út 6. H-7624 Pécs, Hungary
}

E-mail: kurucz@szfki.hu

Received 17 February 2003, accepted for publication 6 May 2003

Published 16 September 2003

Online at stacks.iop.org/JOptB/5/S627

\begin{abstract}
The antilinear operator representation of bipartite pure states of the relative state formulation of quantum mechanics is applied to describe quantum teleportation schemes utilizing an arbitrary pure state as the entangled resource. Bennett type teleportation schemes with nonmaximally entangled pure states are characterized and the notion of 'entanglement matching' is introduced in general. Examples, including a scheme based on coherent-state superposition states of the electromagnetic field, are provided.
\end{abstract}

Keywords: Conditional teleportation, entanglement matching, partially entangled states

\section{Introduction}

In the investigation of quantum teleportation [1] it is interesting to find criteria which have to be met in order to provide faithful teleportation under certain circumstances, including conditional or unconditional schemes. This question is naturally related to the applicability of nonmaximally entangled states as entangled resources, and the classification of possible teleportation schemes. These considerations are interesting even in the case when the entangled resource of the teleportation procedure is a pure state.

Li et al [2] have shown for two-dimensional systems that a nonmaximally entangled resource is applicable for quantum teleportation provided that the measurement made by the sender to carry out teleportation has a nondegenerate eigenstate that 'matches' the state applied as the resource. This question was recently analysed by Agrawal and Pati [3], who included the possibility of entanglement concentration into the argument. The application of nonmaximally entangled states also appeared in the context of coherent-state superpositions $[4,5]$.

A criterion for quantum teleportation was given by Nielsen and Caves [6], who first investigated it in the context of quantum channels or superoperators and who pointed out

3 Author to whom any correspondence should be addressed. the importance of the concept of reversibility of quantum operations from the point of view of teleportation. A quite general criterion for probabilistic teleportation appeared recently [7].

In this paper we demonstrate that the antilinear description of bipartite quantum states [8] provides an excellent framework to describe teleportation generally and to give criteria that have to be fulfilled in order for the teleportation scheme to succeed. There are several advantages of this formalism: it is concise and basis independent. We give a condition for probabilistic teleportation, general enough to describe cases when the Hilbert spaces of the subsystems involved are of different dimensions. A consequence of this condition is the requirement for 'entanglement matching': teleportation succeeds if and only if the state of the entangled resource and the state the sender's projective measurement projects onto 'match' each other. Several examples illustrate the convenience of our general formalism, including an application to teleportation in the subspace spanned by some special coherent states using a nonmaximally entangled coherent channel.

This paper is organized as follows. In section 2, we introduce antilinear operators to describe bipartite pure states. This formalism is than applied in section 3 to a conditional teleportation channel: a simple connection between the quantum operation realized by the channel and 
the antilinear operators describing the entangled resource and the measurement is calculated. In section 4 some examples are given:

(i) the possibility of unconditional schemes is investigated;

(ii) a measurement is presented which 'matches' a general partially entangled state;

(iii) measurement matching in the nonorthogonal basis of certain coherent states is studied.

Section 5 summarizes our results.

\section{Antilinear representation of bipartite pure states}

Consider a bipartite system with two subsystems $A$ and $B$ described by the Hilbert spaces $\mathcal{H}_{A}$ and $\mathcal{H}_{B}$ which may be different (even infinite) dimensional. A general pure state of this bipartite system is normally described by a state vector $|\sigma\rangle_{A B}$ in the Hilbert space $\mathcal{H}_{A} \otimes \mathcal{H}_{B}$. In this section we briefly review a different possibility of describing bipartite pure states, namely the one utilizing antilinear operators [8]. This representation has several advantages, which will prove to be useful in treating problems such as quantum teleportation.

If the two subsystems are entangled, it is meaningless to talk about the state of one subsystem or that of the other. But one can ask what the state $|\psi\rangle_{B}$ of system $B$ is if system $A$ is found to be in state $|\phi\rangle_{A}$ (say, after a measurement performed on system $A$ ). This way of interpreting entangled states forms the base of the so-called relative state approach of Everett [9]. It is easy to verify that the state in question can be obtained by the partial inner product of the state of system $A$ and the bipartite state $|\sigma\rangle_{A B}$ :

$$
|\psi\rangle_{B} \propto{ }_{A}\langle\phi \mid \sigma\rangle_{A B}
$$

The vector on the right-hand side is not normalized. If it is zero then the probability of finding system $A$ in state $|\phi\rangle_{A}$ is zero. Let us define the relative state operator corresponding to the bipartite state $|\sigma\rangle_{A B}$ as an operator mapping $|\phi\rangle_{A}$ to the unnormalized relative state of system $B$ :

$$
L_{\sigma}: \mathcal{H}_{A} \rightarrow \mathcal{H}_{B}, \quad|\phi\rangle_{A} \mapsto{ }_{A}\langle\phi \mid \sigma\rangle_{A B}
$$

Since the scalar product is conjugate linear in its first argument, we have defined an antilinear operator, that is, conjugate linear in its argument.

One may choose (arbitrary) orthonormal bases $|i\rangle_{A}$ in $\mathcal{H}_{A}$ and $|j\rangle_{B}$ in $\mathcal{H}_{B}$ as computational bases. The bipartite pure state $|\sigma\rangle_{A B}$ can then be expanded on the product state basis as $\sum_{i j} c_{i j}|i\rangle_{A} \otimes|j\rangle_{B}$. In the given bases, matrix elements of the corresponding antilinear operator are $L_{j i}^{\sigma}={ }_{B}\left\langle j\left|L_{\sigma}\right| i\right\rangle_{A}=c_{i j}$. Since $L_{\sigma}$ maps the basis of system $A$ to $L_{\sigma}|i\rangle_{A}=\sum_{j} c_{i j}|j\rangle_{B}$, the bipartite state in the argument reads

$$
|\sigma\rangle_{A B}=\sum_{i}|i\rangle_{A} \otimes\left(L_{\sigma}|i\rangle_{A}\right)
$$

This expansion holds for every basis $|i\rangle_{A}$ with the same antilinear operator. Indeed, taking another orthonormal basis
$|k\rangle_{A}^{\prime}=u_{k i}|i\rangle_{A}$, where $u_{k i}$ is a unitary matrix, equation (3) gives the same state

$$
\begin{aligned}
|\sigma\rangle_{A B} & =\sum_{k}|k\rangle_{A}^{\prime} \otimes\left(L_{\sigma}|k\rangle_{A}^{\prime}\right) \\
& =\sum_{i j k} u_{k i}|i\rangle_{A} \otimes\left[L_{\sigma}\left(u_{k j}|j\rangle_{A}\right)\right] \\
& =\sum_{i j} \sum_{k} u_{k i} u_{k j}^{*}|i\rangle_{A} \otimes\left(L_{\sigma}|j\rangle_{A}\right) \\
& =\sum_{i}|i\rangle_{A} \otimes\left(L_{\sigma}|i\rangle_{A}\right) .
\end{aligned}
$$

The fact that $L_{\sigma}$ is antilinear implies the complex conjugation of $u_{k j}$. Note that if the development (3) is done using a linear operator instead, changing the computation basis would result in another bipartite state for the same linear operator, and different linear operators must be defined for different sets of the basis. The antilinear representation, however, gives us an absolute, i.e. basis independent, way to describe bipartite pure states.

As an example, consider the singlet state of two entangled qubits:

$$
\left|\Psi^{-}\right\rangle_{A B}=\frac{1}{\sqrt{2}}\left(\left|\uparrow_{z}\right\rangle_{A} \otimes\left|\downarrow_{z}\right\rangle_{B}-\left|\downarrow_{z}\right\rangle_{A} \otimes\left|\uparrow_{z}\right\rangle_{B}\right) .
$$

The corresponding relative state operator $L_{\Psi^{-}}$(shortly $L$ ) maps the spin-up and spin-down states of system $A$ to $L\left|\uparrow_{z}\right\rangle_{A}=$ $\left|\downarrow_{z}\right\rangle_{B} / \sqrt{2}$ and $L\left|\downarrow_{z}\right\rangle_{A}=-\left|\uparrow_{z}\right\rangle_{B} / \sqrt{2}$ respectively. Indeed, if one measures the spin of system $A$ along the $z$-axis and obtains $\left|\uparrow_{z}\right\rangle_{A}$ (or $\left|\downarrow_{z}\right\rangle_{A}$ ) then the state of system $B$ is $\left|\downarrow_{z}\right\rangle_{B}$ (resp. $\left|\uparrow_{z}\right\rangle_{B}$ ). But if one performs the measurement in the $y$-direction with a result $\left|\uparrow_{y}\right\rangle_{A}=\left(\left|\uparrow_{z}\right\rangle_{A}+\mathrm{i}\left|\downarrow_{z}\right\rangle_{A}\right) / \sqrt{2}$ (or $\left.\left|\downarrow_{y}\right\rangle_{A}=\left(\left|\uparrow_{z}\right\rangle_{A}-\mathrm{i}\left|\downarrow_{z}\right\rangle_{A}\right) / \sqrt{2}\right)$ then the state of system $B$ is proportional to

$$
\begin{aligned}
L\left|\uparrow_{y}\right\rangle_{A} & =L\left[\frac{1}{\sqrt{2}}\left(\left|\uparrow_{z}\right\rangle_{A}+\mathrm{i}\left|\downarrow_{z}\right\rangle_{A}\right)\right] \\
& =\frac{1}{\sqrt{2}}\left(L\left|\uparrow_{z}\right\rangle_{A}-\mathrm{i} L\left|\downarrow_{z}\right\rangle_{A}\right) \\
& =\frac{1}{2}\left(\left|\downarrow_{z}\right\rangle_{B}+\mathrm{i}\left|\uparrow_{z}\right\rangle_{B}\right)=\frac{\mathrm{i}}{\sqrt{2}}\left|\downarrow_{y}\right\rangle_{B}
\end{aligned}
$$

(resp. $L\left|\downarrow_{y}\right\rangle_{A}=-\mathrm{i}\left|\uparrow_{y}\right\rangle_{B} / \sqrt{2}$ ), as expected. In the new basis, the entangled state is expanded according to (3) as

$$
\begin{aligned}
\left|\Psi^{-}\right\rangle_{A B} & =\left|\uparrow_{y}\right\rangle_{A} \otimes\left(L\left|\uparrow_{y}\right\rangle_{A}\right)-\left|\downarrow_{y}\right\rangle_{A} \otimes\left(L\left|\downarrow_{y}\right\rangle_{A}\right) \\
& =\frac{\mathrm{i}}{\sqrt{2}}\left(\left|\uparrow_{y}\right\rangle_{A} \otimes\left|\downarrow_{y}\right\rangle_{B}-\left|\downarrow_{y}\right\rangle_{A} \otimes\left|\uparrow_{y}\right\rangle_{B}\right) .
\end{aligned}
$$

The relative state operator formalism provides us with a complete description of pure bipartite states. In general, the set $\mathcal{C}_{A B}$ of bounded antilinear operators $L: \mathcal{H}_{A} \rightarrow \mathcal{H}_{B}$ having a finite norm forms a Hilbert space isomorphic to $\mathcal{H}_{A} \otimes \mathcal{H}_{B}$ [8]:

$$
\mathcal{C}_{A B}=\left\{L: \mathcal{H}_{A} \rightarrow \mathcal{H}_{B} \text { bounded antilinear } \mid \operatorname{Tr}\left(L^{\dagger} L\right)<\infty\right\} .
$$

The norm on $\mathcal{C}_{A B}$ is derived from the scalar product $\left(L, L^{\prime}\right)=$ $\operatorname{Tr}\left(L^{\prime \dagger} L\right)$, which is conjugate linear in the first argument. The adjoint of an antilinear operator is defined by the relation $\langle f \mid L e\rangle=\left\langle L^{\dagger} f \mid e\right\rangle^{*}$, for all $|e\rangle \in \mathcal{H}_{A}$ and $|f\rangle \in \mathcal{H}_{B}$. The adjoint corresponds to the transpose of the matrix of $L$. Thus every pure bipartite state $|\sigma\rangle_{A B}$ can uniquely be described by an antilinear operator $L_{\sigma} \in \mathcal{C}_{A B}$ such that $\operatorname{Tr}\left(L_{\sigma}^{\dagger} L_{\sigma}\right)=1$. 
An operator description of entanglement matching in quantum teleportation

The relative state operator formalism proves to be of the utmost convenience in describing partial traces of the bipartite system, and thus characterizing entanglement. The reduced density matrices, that is, the partial traces of the bipartite state in one of the subsystems, can be expressed as

$$
\begin{gathered}
\varrho_{A}=\operatorname{Tr}_{B}|\sigma\rangle_{A B A B}\langle\sigma|=L_{\sigma}^{\dagger} L_{\sigma}, \\
\varrho_{B}=\operatorname{Tr}_{A}|\sigma\rangle_{A B A B}\langle\sigma|=L_{\sigma} L_{\sigma}^{\dagger}
\end{gathered}
$$

respectively. Notice the extreme simplicity of the above formulae, which may be regarded as one of the main advantages of this representation.

Equation (9) enables us to easily characterize maximally entangled states. They are those states whose density operator has a partial trace proportional to identity. Since maximally entangled states exist only in finite dimensional Hilbert spaces, suppose here that $\mathcal{H}_{A}$ and $\mathcal{H}_{B}$ are of the same finite dimensionality $N$. Thus the state in (3) is maximally entangled if and only if $L L^{\dagger}=N^{-1} I_{B}$ and $L^{\dagger} L=N^{-1} I_{A}$. This is equivalent to the fact that $\sqrt{N} L$ is antiunitary. (An antilinear operator $A$ is called antiunitary if $A^{\dagger} A$ and $A A^{\dagger}$ are the identity operators of the respective state spaces.) Maximally entangled states are exactly those described by antiunitary operators in the antilinear operator formalism.

\section{Conditions for quantum teleportation}

In this section we present an application of the antilinear description of bipartite pure states introduced in section 2 for quantum teleportation [10].

Suppose that system $A$ prepared in the unknown input state $|\phi\rangle_{A}$ is to be teleported, and systems $B$ and $C$ are in the partially entangled shared state:

$$
|\sigma\rangle_{B C}=\sum_{i}|i\rangle_{B} \otimes\left(L|i\rangle_{B}\right)
$$

which is described by the antilinear map $L \in \mathcal{C}_{B C}$. Systems $A$ and $B$ are located at Alice who performs a joint measurement on them. Suppose that the state of the compound system $A B$ is projected onto the state

$$
\left|\sigma_{q}\right\rangle_{A B}=\sum_{i}\left(L_{q}|i\rangle_{B}\right) \otimes|i\rangle_{B},
$$

where $L_{q} \in \mathcal{C}_{B A}$. Note that the basis $|i\rangle_{B}$ in the description of the shared state and of the measurement outcome are common. In the followings, we will regard only this outcome, so our teleportation scheme will be probabilistic.

Now we will calculate the teleportation channel. By this we mean a function $f_{q}: \mathcal{H}_{A} \longmapsto \mathcal{H}_{C}$ that relates the input state $|\phi\rangle_{A}$ and the state of system $C$ after the measurement. We may also include in the formalism the unitary operator $U_{q}$ applied by Bob. At the beginning, the three systems are in the state $|\phi\rangle_{A} \otimes|\sigma\rangle_{B C}$. The probability of the outcome under consideration is given by

$$
\begin{aligned}
p_{q}\left(|\phi\rangle_{A}\right) & =\|\left[\left(\left|\sigma_{q}\right\rangle_{A B A B}\left\langle\sigma_{q}\right|\right) \otimes I_{C}\right]\left(|\phi\rangle_{A} \otimes|\sigma\rangle_{B C}\right) \|^{2} \\
& =\| L L_{q}^{\dagger}|\phi\rangle_{A} \|^{2} .
\end{aligned}
$$

On condition that the measurement yields the outcome $q$, the state of system $C$ can be obtained by similar calculation, so the teleportation channel for the outcome $q$ is

$$
f_{q}: \mathcal{H}_{A} \longmapsto \mathcal{H}_{C}, \quad f_{q}\left(|\phi\rangle_{A}\right)=\frac{U_{q} L L_{q}^{\dagger}|\phi\rangle_{A}}{\| L L_{q}^{\dagger}|\phi\rangle_{A} \|} .
$$

If the input state is given by the density operator $\rho_{\text {in }}$ then the output state is

$$
\rho_{\text {out }}=\frac{U_{q} L L_{q}^{\dagger} \rho_{\mathrm{in}} L_{q} L^{\dagger} U_{q}^{\dagger}}{\operatorname{Tr}_{A}\left(L_{q} L^{\dagger} L L_{q}^{\dagger} \rho_{\mathrm{in}}\right)} .
$$

In a faithful teleportation scheme, the output state equals the input state in the sense that $f_{q}$ is a unitary isomorphism between $\mathcal{H}_{A}$ and $\mathcal{H}_{C}$. Thus, Bob can apply a unitary transformation to restore the input state. Such a scheme is called unitarily reversible and has been studied in [6]. In our scheme, however, $f_{q}$ may not even be linear due to the fact that post selection of the measurement outcome $q$ implies a normalization factor in (13). Therefore, we adopt a more general definition here. We call the channel $f_{q}$ reversible if it is injective, that is, for different input state $|\phi\rangle_{A}$ the corresponding output state $f_{q}\left(|\phi\rangle_{A}\right)$ is different. The output of such a reversible teleportation channel can be transformed (theoretically) into the original input state by applying the inverse of $f_{q}$. This nonunitary transformation can be envisaged as a probabilistic method [11], or can be approximated by completely positive maps [12].

One may easily verify that our condition of reversibility is equivalent to the invertibility of the linear operator $L L_{q}^{\dagger}: \mathcal{H}_{A} \rightarrow$ $\mathcal{H}_{C}$. It is proved in [10] that for such a reversible teleportation channel the following three statements are equivalent:

(i) $f_{q}$ is linear, that is, $f_{q}\left(\alpha_{1}\left|\phi_{1}\right\rangle+\alpha_{2}\left|\phi_{2}\right\rangle\right)=\alpha_{1} f_{q}\left(\left|\phi_{1}\right\rangle\right)+$ $\alpha_{2} f_{q}\left(\left|\phi_{2}\right\rangle\right)$,

(ii) the probability $p_{q}\left(|\phi\rangle_{A}\right)$ of the outcome $q$ defined in (12) is independent of the input state $|\phi\rangle_{A}$, that is, Alice learns nothing from her measurement,

(iii) $f_{q}$ is unitarily reversible.

Given a partially entangled shared state, the question naturally arises: what are the conditions the joint measurement has to fulfil in order to obtain a unitarily reversible teleportation channel? Suppose that the Hilbert spaces considered below are finite dimensional, namely $\operatorname{dim} \mathcal{H}_{A} \leqslant \operatorname{dim} \mathcal{H}_{B}=\operatorname{dim} \mathcal{H}_{C}$. Let the shared state $|\sigma\rangle_{B C}$ be described by an invertible conjugate linear operator $L$. The criterion of invertibility in finite dimensions means that every coefficient in its Schmidt decomposition is nonzero. (If it is not so then we can restrict the domain of $L$ and regard only the orthogonal complement of its kernel instead of $\mathcal{H}_{B}$.) Let $i_{A C}$ be a unitary embedding of $\mathcal{H}_{A}$ into $\mathcal{H}_{C}$ so that we can compare the states of systems $A$ and $C$. The condition for unitary reversibility reads

$$
\frac{1}{\sqrt{p_{q}}} U_{q} L L_{q}^{\dagger}=i_{A C}
$$

which also guarantees that the probability $p_{q}$ is independent of the input state $|\phi\rangle_{A}$. From this we conclude that obtaining 
a measurement outcome $q$ corresponding to a projection onto the state described by the antilinear operator

$$
L_{q}=\sqrt{p_{q}} i_{A C}^{\dagger} U_{q} L^{-1 \dagger}
$$

provides us with the opportunity of fidelity 1 conditional teleportation. The appropriate recovering unitary transformation to be applied by Bob is $U_{q}$. For a given shared state, the matrix of (16) will be calculated in sections 4.2 and 4.3.

Although $p_{q}$ in (12) depends on $L_{q}$, this can be resolved by the fact that $L_{q}$ has a norm $\operatorname{Tr}_{B}\left(L_{q}^{\dagger} L_{q}\right)=1$. Then we obtain

$$
p_{q}=\left[\operatorname{Tr}_{B}\left(L^{-1} \Pi_{q} L^{-1^{\dagger}}\right)\right]^{-1},
$$

where $\Pi_{q}=U_{q}^{\dagger} i_{A C} i_{A C}^{\dagger} U_{q}$ is a projection onto $U_{q}^{\dagger}\left[\operatorname{Ran} i_{A C}\right]$, and Ran denotes the range of the operator. $\Pi_{q}$ is the identity of $\mathcal{H}_{C}$ only if $\mathcal{H}_{A}$ and $\mathcal{H}_{C}$ have the same dimensions, which is often considered in teleportation. In section 4.1, teleportation from a smaller state space to a larger one is demonstrated.

Thus not every possible measurement outcome allows teleportation, only those described by (16) (for some arbitrary $U_{q}$ ). The measurement and the shared state should be 'matched' to each other. This can be regarded as a generalization of 'entanglement matching' introduced in [2].

Note that in the above description of teleportation it is assumed that the state of the shared pair is pure and a nondegenerate projective measurement is performed on Alice's side. To generalize the scheme, one should first consider degenerate projective measurements, that is, when the state of systems $A$ and $B$ is projected onto a two- (or more) dimensional subspace. Suppose that for such a degenerate measurement outcome $q$ this subspace is spanned by the orthonormal eigenvectors $\left|\sigma_{q, i}\right\rangle_{A B}$ (with $i=1,2, \ldots$ ). The corresponding antilinear operators are denoted by $L_{q, i} \in$ $\mathcal{C}_{B A}$. To calculate the effect of the measurement, one has to apply the projector $P_{q}=\sum_{i}\left|\sigma_{q, i}\right\rangle_{A B A B}\left\langle\sigma_{q, i}\right|$. Utilizing the unnormalized version of (14), this implies that the output state is the following mixed state:

$$
\varrho_{\text {out }}=\frac{\sum_{i} U_{q} L L_{q, i}^{\dagger} \varrho_{\text {in }} L_{q, i} L^{\dagger} U_{q}^{\dagger}}{\operatorname{Tr}\left[\left(\sum_{k} L_{q, k} L^{\dagger} L L_{q, k}^{\dagger}\right) \varrho_{\text {in }}\right]} .
$$

Now the generalization to POVM (positive operatorvalued measure) measurements is straightforward since every such measurement can be traced back to a projective measurement by extending the state space. This can easily be done by enlarging system $B$ and performing a projective measurement on system $A$ and the extended system $B^{\prime}$. Since we are not interested in the final state of system $B$, no tracing out to the extra system is needed. However, attention should be paid to the point that the inverse of $L$ is taken with respect to the restricted system $B$.

If, in addition, the shared resource is not pure but a convex combination of the pure states $\left|\sigma_{j}\right\rangle_{B C}$ (given by the antilinear operators $L_{j} \in \mathcal{C}_{B C}$ ) then the output will also be the convex combination of results corresponding to each pure state $\left|\sigma_{j}\right\rangle_{B C}$ :

$$
\varrho_{\text {out }}=\mathcal{N} \sum_{i j} w_{j} U_{q} L_{j} L_{q, i}^{\dagger} \varrho_{\text {in }} L_{q, i} L_{j}^{\dagger} U_{q}^{\dagger},
$$

where $\mathcal{N}$ is a normalizing factor and $w_{j}$ are the nonnegative weights of the convex combination summing up to 1 .

\section{Examples}

In the following, we investigate some special examples demonstrating the applicability of the general considerations of the previous section.

\subsection{Unconditional schemes}

Let us consider the case when dimensions of systems $A, B$ and $C$ are the same, so $\Pi_{q}$ is the identity operator. One of the interesting results is that all the matching outcomes have the same probability

$$
p=\left[\operatorname{Tr}_{B}\left(\left(L^{\dagger} L\right)^{-1}\right)\right]^{-1}
$$

and this probability depends on the shared state only. Since there are at most $\left(\operatorname{dim} \mathcal{H}_{A}\right)\left(\operatorname{dim} \mathcal{H}_{B}\right)$ possible outcomes for a projective measurement, faithful unconditional teleportation (always succeeding with fidelity 1 ) is possible only with shared states satisfying $p_{q}=1 /\left(\operatorname{dim} \mathcal{H}_{A} \operatorname{dim} \mathcal{H}_{B}\right)$. Not surprisingly, these shared states are maximally entangled [6].

Dimensionalities need not, however, be the same for all systems. As an example, suppose that system $A$ is a two-state system, $B$ and $C$ are three-state ones, and let the shared state be maximally entangled:

$$
\sigma=\frac{1}{\sqrt{3}}\left(|00\rangle_{B C}+|11\rangle_{B C}+|22\rangle_{B C}\right) .
$$

If Alice measures an observable having the following nondegenerate eigenstates, it will implement a fidelity 1 unconditional teleportation:

$$
\begin{aligned}
& \sigma_{1,2}=\left(|00\rangle_{A B} \pm|11\rangle_{A B}\right) / \sqrt{2}, \\
& \sigma_{3,4}=\left(|01\rangle_{A B} \pm|12\rangle_{A B}\right) / \sqrt{2}, \\
& \sigma_{5,6}=\left(|02\rangle_{A B} \pm|10\rangle_{A B}\right) / \sqrt{2} .
\end{aligned}
$$

Every outcome has a probability of $1 / 6$. If Alice finds her systems $A$ and $B$ in state $\left|\sigma_{3}\right\rangle_{A B}$, for example, the basis $|0\rangle_{A},|1\rangle_{A}$ of system $A$ is teleported into the basis $|1\rangle_{C},|2\rangle_{C}$ of system $C$, which spans a two-dimensional subspace of the $\mathcal{H}_{C}$. This way, teleportation from a system with smaller state space to a system with larger state space can be realized.

This example can be generalized easily. Let $A$ be an $n$-state system and $B$ and $C$ be $m$-state ones $(n<m)$. The measurement basis can be indexed by the numbers $i=$ $0, \ldots, n-1$ and $j=0, \ldots, m-1$, and the basis has the form

$$
\sigma_{(i, j)}=\sum_{k=0}^{n-1} \varepsilon^{j k}|k\rangle_{A}|(k+i) \bmod m\rangle_{B},
$$

where $\varepsilon=\exp (2 \pi \mathrm{i} / n)$ is the complex $n$th root of 1 . This can be regarded as an extension of Bell basis on systems having state spaces of different dimensionality. These states are not maximally entangled, since their partial trace over system $A$ is not proportional to identity but to $\Pi_{(i, j)}$. 


\subsection{Partially entangled shared states}

We give another example for measurement matching in the case of a partially entangled shared state which is given by the following matrix in convenient bases $|i\rangle_{B}$ and $|j\rangle_{C}$ :

$$
L=\left(\begin{array}{ccc}
\alpha_{1} & & \\
& \ddots & \\
& & \alpha_{n}
\end{array}\right), \quad \sigma=\sum_{i=1}^{n} \alpha_{i}|i\rangle_{B}|i\rangle_{C}
$$

where all $\alpha_{i}$ are nonzero and $n=\operatorname{dim} \mathcal{H}_{A}=\operatorname{dim} \mathcal{H}_{B}=$ $\operatorname{dim} \mathcal{H}_{C}$. Consider a Schmidt decomposition, for instance, with the convenient bases being formed from the Schmidt vectors. We obtain from (16) that a matching measurement outcome can be represented by the state described by

$$
\begin{aligned}
L_{1} & =\left(\sum_{i} \frac{1}{\left|\alpha_{i}\right|^{2}}\right)^{-1 / 2}\left(\begin{array}{ccc}
1 / \alpha_{1}^{*} & & \\
& \ddots & \\
& & 1 / \alpha_{n}^{*}
\end{array}\right), \\
\sigma_{1} & =\left(\sum_{i} \frac{1}{\left|\alpha_{i}\right|^{2}}\right)^{-1 / 2} \sum_{i} \frac{1}{\alpha_{i}^{*}}|i\rangle_{A}|i\rangle_{B} .
\end{aligned}
$$

The basis $|i\rangle_{A}$ in $\mathcal{H}_{A}$ should be chosen to correspond to the isomorphism $i_{A C}$ and the unitary transformation $U_{q}$. Thus if Alice measures an observable with an eigenstate equal to $\sigma_{1}$ then this measurement outcome enables us to carry out a conditional teleportation. The probability of this outcome is

$$
p=\left(\sum_{i} \frac{1}{\left|\alpha_{i}\right|^{2}}\right)^{-1} .
$$

A similar result was found for two dimensions in [3]. If the shared state is $|\sigma\rangle_{B C}=\alpha_{0}|0\rangle_{A}|0\rangle_{B}+\alpha_{1}|1\rangle_{A}|1\rangle_{B}$, then the state in (27) reduces to

$$
\left|\sigma_{1}\right\rangle_{A B}=\mathrm{e}^{\mathrm{i} \phi} \alpha_{1}|0\rangle_{A}|0\rangle_{B}+\mathrm{e}^{-\mathrm{i} \phi} \alpha_{0}|1\rangle_{A}|1\rangle_{B},
$$

where $\phi=\arg \alpha_{0}-\arg \alpha_{1}$ is the difference of the complex phases of the two coefficients $\alpha_{0}$ and $\alpha_{1}$. The state of the shared resource and that of the matching measurement outcome have the same structure. Therefore, their von Neumann reduced entropies are equal. In higher dimensions, however, our results show that the entropies do not coincide.

\subsection{Entanglement matching in coherent basis}

In our last example, we consider simple bosonic systems, e.g. modes of the electromagnetic field. We regard only a twodimensional subspace of its infinite dimensional Hilbert space, which is spanned by the nonorthogonal basis consisting of the coherent states $|\alpha\rangle$ and $|-\alpha\rangle$. Our objective is to faithfully teleport a superposition of these two coherent states using the following two-mode partially entangled resource:

$$
|\sigma\rangle_{B C}=N_{\eta}^{-1 / 2}\left(\cos \eta|\alpha\rangle_{B}|\alpha\rangle_{C}-\sin \eta|-\alpha\rangle_{B}|-\alpha\rangle_{C}\right),
$$

where $N_{\eta}=1-\sin 2 \eta \mathrm{e}^{-4|\alpha|^{2}}$ is a normalization factor. A similar problem was investigated in $[4,5]$. Choosing $\sin 2 \omega=$ $\langle\alpha \mid-\alpha\rangle=\mathrm{e}^{-2|\alpha|^{2}}$ and $N_{\omega}=\cos ^{2} 2 \omega$, the following vectors form an orthonormal basis in the subspace under consideration:

$$
\begin{aligned}
\left|\psi_{+}\right\rangle & =N_{\omega}^{-1 / 2}(\cos \omega|\alpha\rangle-\sin \omega|-\alpha\rangle), \\
\left|\psi_{-}\right\rangle & =N_{\omega}^{-1 / 2}(-\sin \omega|\alpha\rangle+\cos \omega|-\alpha\rangle) .
\end{aligned}
$$

In this orthonormal basis, the shared state (30) can be expanded as

$$
|\sigma\rangle_{B C}=A\left|\psi_{+}\right\rangle_{B}\left|\psi_{+}\right\rangle_{C}+B\left|\psi_{-}\right\rangle_{B}\left|\psi_{-}\right\rangle_{C}
$$

$$
+C\left(\left|\psi_{+}\right\rangle_{B}\left|\psi_{-}\right\rangle_{C}+\left|\psi_{-}\right\rangle_{B}\left|\psi_{+}\right\rangle_{C}\right)
$$

with

$$
\begin{gathered}
A=N_{\eta}^{-1 / 2}\left(\cos \eta \cos ^{2} \omega-\sin \eta \sin ^{2} \omega\right), \\
B=N_{\eta}^{-1 / 2}\left(\cos \eta \sin ^{2} \omega-\sin \eta \cos ^{2} \omega\right), \\
C=N_{\eta}^{-1 / 2}(\cos \eta-\sin \eta) \sin \omega \cos \omega .
\end{gathered}
$$

Note that coefficient $C$ of the cross terms becomes exponentially small if the coherent amplitude $\alpha$ is large and it vanishes for $\eta=\pi / 4$. This later corresponds to maximal entanglement. However, we will not neglect it in our calculations. The matrix of the antilinear operator describing (32) is

$$
L=\left(\begin{array}{ll}
A & C \\
C & B
\end{array}\right) .
$$

The probability (17) of a matching outcome reads

$$
p=\frac{\sin ^{2} 2 \eta \cos ^{4} 2 \omega}{4\left(1-\sin 2 \eta \sin ^{2} 2 \omega\right)^{2}} .
$$

Using (16), a possible antilinear operator corresponding to the matching outcome is given by the antilinear operator

$$
L_{1}=\sqrt{p} L^{-1 \dagger}=\left(\begin{array}{cc}
B & -C \\
-C & A
\end{array}\right),
$$

which describes the 'matching' entangled state

$\left|\sigma_{1}\right\rangle_{A B}=X|\alpha\rangle_{A}|\alpha\rangle_{B}+Y|-\alpha\rangle_{A}|-\alpha\rangle_{B}$

$$
+Z\left(|\alpha\rangle_{A}|-\alpha\rangle_{B}+|-\alpha\rangle_{A}|\alpha\rangle_{B}\right)
$$

with coefficients

$$
\begin{aligned}
& X=N_{\eta}^{-1 / 2} N_{\omega}^{-1}\left(\cos \eta \sin ^{2} \omega-\sin \eta\right), \\
& Y=N_{\eta}^{-1 / 2} N_{\omega}^{-1}\left(\cos \eta-\sin \eta \sin ^{2} \omega\right), \\
& Z=N_{\eta}^{-1 / 2} N_{\omega}^{-1}(\cos \eta-\sin \eta) \sin 2 \omega .
\end{aligned}
$$

Thus a fidelity 1 teleportation occurs on condition that Alice projects her two systems onto this latter entangled state.

\section{Conclusions}

We found it useful in the description of teleportation to represent a bipartite pure state with the antilinear operator which determines the relative state representation of the state. We arrived at a simple connection between the teleportation channel and the antilinear operators describing (i) the joint measurement performed by the sender and (ii) the entangled resource shared by the parties. Characterizing all conditional teleportation schemes, we showed that the joint measurement has to 'match' the partially entangled shared state in order to achieve fidelity 1 conditional teleportation. The presented formalism allows us to specify all measurement outcomes that match a given partially entangled pure shared state. Three examples were presented to illustrate the power of this relative state operator formalism. First, we outlined a proof of the fact that only maximal entanglement can play role in unconditional teleportation. We gave a possible measurement outcome for a general pure partially entangled shared state. Finally, we solved the problem of entanglement matching for a partially entangled optical Schrödinger cat type state as the shared resource. 


\section{Acknowledgment}

Our research was partially supported by the National Research Fund of Hungary (OTKA) under contract no T034484.

\section{References}

[1] Bennett C H, Brassard G, Crépeau C, Jozsa R, Peres A and Wootters W K 1993 Phys. Rev. Lett. 701895

[2] Li W-L, Li C-F and Guo G-C 2000 Phys. Rev. A 61034301

[3] Agrawal P and Pati A K 2002 Phys. Lett. A 30512
[4] Jeong H, Kim M S and Lee J 2001 Phys. Rev. A 64052308

[5] Zheng Y-Z, Gu Y-J and Guo G-C 2003 J. Opt. B: Quantum Semiclass. Opt. 529

[6] Nielsen M A and Caves C M 1997 Phys. Rev. A 552547

[7] Li C, Song H-S and Luo Y-X 2002 Phys. Lett. A 297121

[8] Arens R and Varadarajan V S 2000 J. Math. Phys. 41638

[9] Everett H III 1957 Rev. Mod. Phys. 29454

[10] Kurucz Z, Koniorczyk M and Janszky J 2001 Fortschr. Phys. 491019

[11] Bergou J A, Hillery M and Sun Y 2000 J. Mod. Opt. 47487

[12] Řeháček J, Hradil Z, Fiurášek J and Brukner Č 2001 Phys. Rev. A 64 060301(R) 